\title{
Erratum to: Conversion of Uric Acid into Ammonium in Oil-Degrading Marine Microbial Communities: a Possible Role of Halomonads
}

\author{
Christoph Gertler ${ }^{1,11} \cdot$ Rafael Bargiela ${ }^{2}$. Francesca Mapelli ${ }^{3}$ - Xifang Han ${ }^{4}$. \\ Jianwei Chen ${ }^{4}$ Tran Hai ${ }^{1}$. Ranya A. Amer ${ }^{5}$ Mouna Mahjoubi ${ }^{6}$ - Hanan Malkawi ${ }^{7}$. \\ Mirko Magagnini ${ }^{8}$ - Ameur Cherif ${ }^{6}$ - Yasser R. Abdel-Fattah ${ }^{5} \cdot$ Nicolas Kalogerakis $^{9}$. \\ Daniele Daffonchio $^{3,10} \cdot$ Manuel Ferrer $^{2} \cdot$ Peter N. Golyshin ${ }^{1}$
}

Published online: 12 June 2015

(C) Springer Science+Business Media New York 2015

\section{Erratum to: Microb Ecol (2015) \\ DOI 10.1007/s00248-015-0606-7}

The original article unfortunately contained errors in the author's affiliations.

Francesca Mapelli is exclusively affiliated to the Department of Food, Environmental and Nutritional Sciences (DeFENS), University of Milan. The correct version of the author's affiliations is presented above.

The online version of the original article can be found at http://dx.doi.org/ 10.1007/s00248-015-0606-7.

Christoph Gertler

christoph.gertler@fli-bund.de

1 School of Biological Sciences, Environment Centre Wales, Bangor University, LL57 2UW Bangor, Gwynedd, UK

2 Consejo Superior de Investigaciones Científicas (CSIC), Institute of Catalysis, 28049 Madrid, Spain

3 Department of Food, Environmental and Nutritional Sciences (DeFENS), University of Milan, via Celoria 2, 20133 Milan, Italy

4 BGI Tech Solutions Co., Ltd, Main Building, Beishan Industrial Zone, Yantian District, Shenzhen 518083, China

5 Genetic Engineering and Biotechnology Research Institute, City for Scientific Research \& Technology Applications, Alexandria, Egypt
6 Highe Higher Institute for Biotechnology, Biotechpole of Sidi Thabet, University of Manouba, LR11ES31, 2020 Sidi Thabet, Ariana, Tunisia

7 Deanship of Research \& Doctoral Studies, Hamdan Bin Mohammad Smart University, Dubai, United Arab Emirates

8 EcoTechSystems Ltd., Ancona, Italy

9 School of Environmental Engineering, Technical University of Crete, Chania, Greece

10 BESE Division, King Abdullah University of Science and Technology, Thuwal 23955-6900, Kingdom of Saudi Arabia

11 Friedrich-Loeffler-Institut - Federal Research Institute for Animal Health, Institute of Novel and Emerging Diseases, Südufer 10, 17493 Greifswald, Insel Riems, Germany 